Rechtsmedizin $2022 \cdot 32: 201-206$

https://doi.org/10.1007/s00194-021-00516-2

Angenommen: 8. Juni 2021

Online publiziert: 7. Juli 2021

(c) Der/die Autor(en) 2021

\title{
Zwei Fälle kardialer Sarkoidose als Ursache des plötzlichen Herztodes
}

\author{
Clara-Sophie Schwarz ${ }^{1}$ Cleo-Aron Weis ${ }^{2} \cdot$ Alexander Marx $^{2} \cdot$ Tanja Germerott $^{1}$. \\ Cleo Walz ${ }^{1}$ \\ ${ }^{1}$ Institut für Rechtsmedizin, Universitätsmedizin Mainz, Mainz, Deutschland \\ ${ }^{2}$ Pathologisches Institut, Universitätsmedizin Mannheim, Mannheim, Deutschland
}

\section{Zusammenfassung}

Eine der primären Aufgaben der Rechtsmedizin ist die Aufklärung unerwarteter Todesfälle. Speziell das Erkennen seltener Todesursachen setzt ein fundiertes Wissen um die typischen makromorphologischen Befunde sowie das Einleiten und die Interpretation entsprechend geeigneter Ergänzungsuntersuchungen, etwa histologischer Verfahren, voraus. Wichtige Differenzialdiagnosen müssen bekannt sein, damit die Todesursache mit vertretbarem diagnostischem Aufwand festgestellt werden kann.

In der vorliegenden Arbeit werden charakteristische makroskopische und mikroskopische Befunde der kardialen Sarkoidose (oder granulomatösen Myokarditis) und deren Abgrenzung zur Riesenzellmyokarditis anhand von 2 Fällen demonstriert. In beiden Fällen gab der plötzliche Tod einer erwachsenen Person mittleren Alters Anlass zu einer gerichtlichen Obduktion, die jeweils zur Diagnose einer kardialen Sarkoidose führte. Die unterschiedlichen Krankheitsverläufe und Befunde werden unter Berücksichtigung einschlägiger wissenschaftlicher Literatur diskutiert. Durch eine sorgfältige Erhebung der Vorgeschichte und der makromorphologischen Befunde kann die Verdachtsdiagnose einer kardialen Sarkoidose unmittelbar nach der Obduktion geäußert und durch histopathologische Untersuchungen bestätigt werden. Es wird deutlich, dass eine interdisziplinäre Fallarbeit bei seltenen Erkrankungen wie der kardialen Sarkoidose eine schnelle Diagnose ermöglicht.

\section{Schlüsselwörter}

Riesenzellmyokarditis · Granulomatöse Myokarditis · Nichtinfektiöse Myokarditis · Plötzliche Todesfälle · Rechtsmedizinische Obduktion

\section{Einführung in das Thema}

Die medizinische Aufklärung von plötzlichen Todesfällen aus natürlicher Ursache gehört zum rechtsmedizinischen Arbeitsalltag. Speziell die Abklärung von seltenen Todesursachen erfordert eine profunde Kenntnis typischer makromorphologischer Befunde und möglicher klinischer Manifestationen. Nur so ist es möglich, schon während der Obduktion unter Berücksichtigung der Vorgeschichte zu einer Arbeitsdiagnose zu gelangen, sodass sinnvolle ergänzende Untersuchungen eingeleitet werden können.
Die Sarkoidose ist eine systemische Erkrankung, die neben anderen Organsystemen auch das Herz betreffen kann. Eine kardiale Sarkoidose oder, wenn der Nachweis sarkoidosetypischer Veränderungen in anderen Organen nicht gelingt, eine idiopathische granulomatöse Myokarditis kann sich in einem plötzlichen Herztod manifestieren, ohne vorher symptomatisch gewesen zu sein. Die wichtigste Differenzialdiagnose der kardialen Sarkoidose ist die Riesenzellmyokarditis [1, 2], bei der es sich ebenfalls um eine nichtinfektiöse Myokarditis handelt.

Im Folgenden werden 2 Fälle von plötzlichem Herztod vorgestellt. Die postmor- 

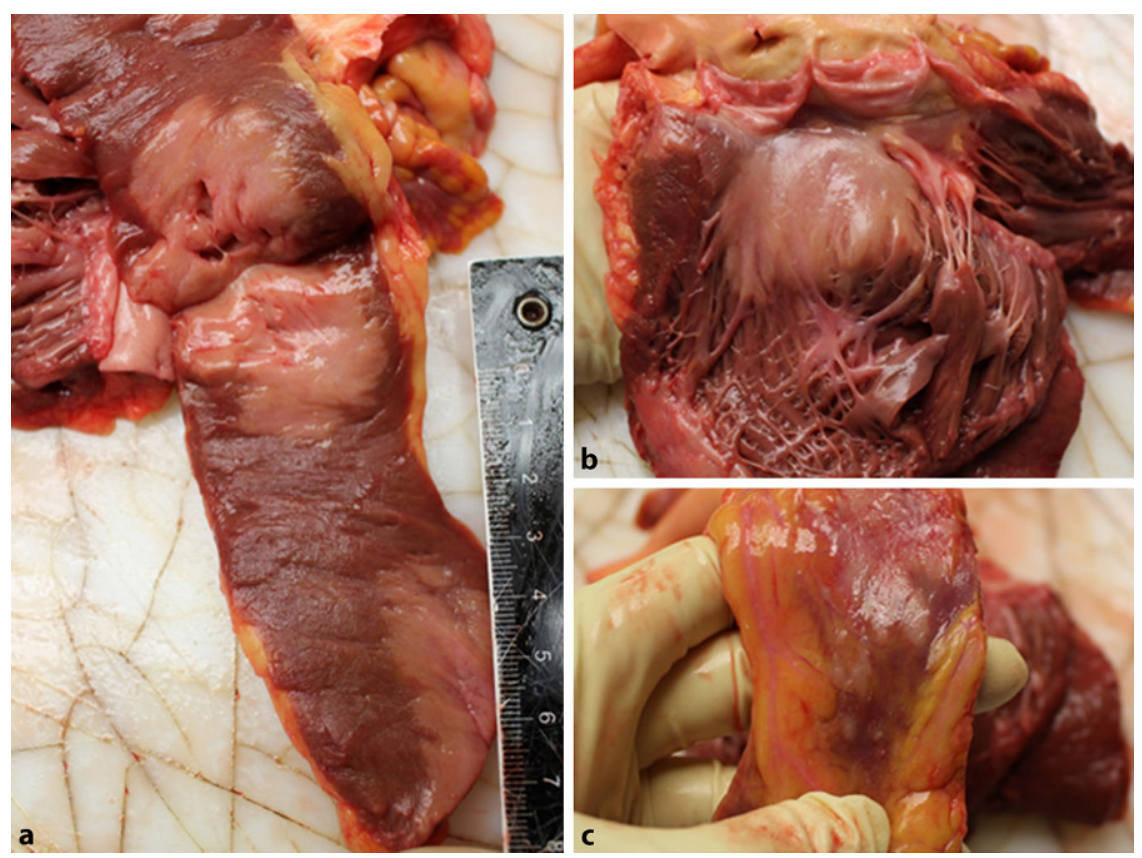

Abb. 1 \ Übersicht des präparierten Herzens mit Blick in den linken Ventrikel. Die Läsionen des Myokards (a) sind durch das Endo- (b) und Epikard (c) sichtbar. Die innere Besichtigung erbrachte einen auffälligen Herzbefund, der die Annahme eines akuten Herzpumpversagens nahelegte

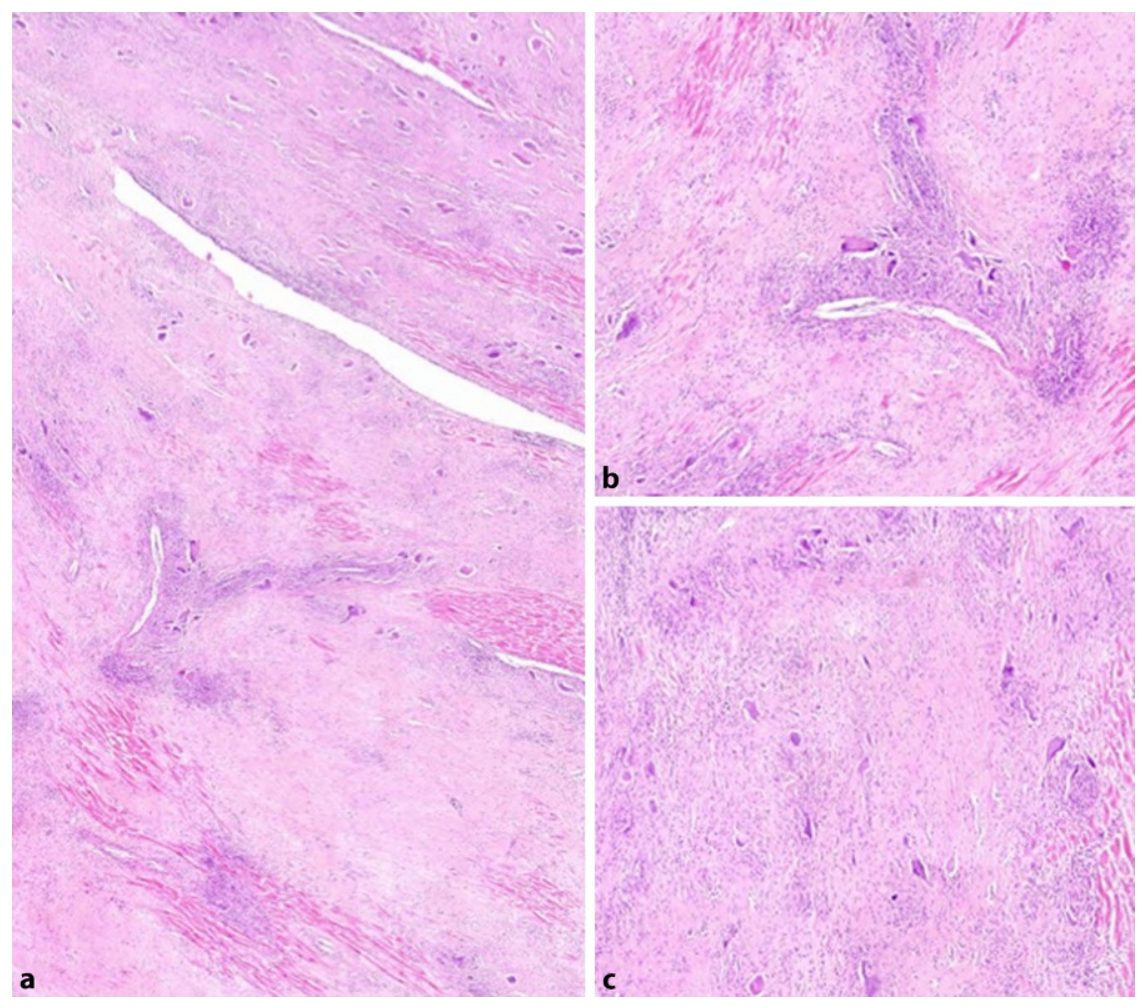

Abb. 2 ム Histologische Darstellung von Fall 1. HE-gefärbtes Gewebe in der Übersicht (a) (Vergrößerung $5 \mathbf{x}$ ) und Vergrößerung $(\mathbf{b}, \mathbf{c})$ (Vergrößerung 10x) mit lymphohistozytärer Infiltration, Riesenzellen und epitheloiden Granulomen. Im Hintergrund ist eine Fibrose zu erkennen talen Diagnosewege werden anhand der einschlägigen wissenschaftlichen Literatur beschrieben. Vor allem werden differentialdiagnostische Überlegungen zur Abgrenzung der kardialen Sarkoidose von der Riesenzellmyokarditis aufgezeigt.

\section{Falldarstellungen}

\section{Fall 1}

\section{Anamnese}

Zur Obduktion gelangte die Leiche einer 55 Jahre alt gewordenen Frau. Nach Angaben ihres Ehemannes habe sie im Bett gelegen und am frühen Morgen plötzlich um Luft gerungen. Nachdem die Frau das Bewusstsein verloren habe, habe der Ehemann unmittelbar mit Wiederbelebungsmaßnahmen begonnen, welche durch den Notarzt fortgesetzt worden seien. Schließlich seien die Maßnahmen vor Ort erfolglos eingestellt worden. Nach den Angaben des Ehemannes seien keine relevanten Vorerkrankungen bekannt gewesen. Die Verstorbene habe sich längere Zeit nicht in ärztlicher Behandlung befunden. Ein Allgemeinmediziner, welchem die Verstorbene persönlich nicht bekannt war, bescheinigte eine ungeklärte Todesart und verständigte die Polizei. Auf der Todesbescheinigung wurde vermerkt, dass die Verstorbene vor ihrem Tod keine gesundheitlichen Beschwerden angegeben habe. Als Vorerkrankung wurde auf der Todesbescheinigung lediglich eine Eisenmangelanämie dokumentiert. Die Leichenschau vor Ort ergab keine Hinweise auf eine Gewalteinwirkung.

\section{Obduktionsbefund}

Bei der äußeren Besichtigung der Leiche $(169 \mathrm{~cm}, 77 \mathrm{~kg})$ waren an postentzündliche Hyperpigmentierung erinnernde, fleckige Hautveränderungen im Gesicht, an der Brust, an den Armen und an den Beinen zu erkennen. Bei der inneren Besichtigung wurde eine linksbetonte konzentrische Herzhypertrophie (Herzgewicht $438 \mathrm{~g}$ ) festgestellt. Besonders auffällig waren umschriebene großfleckige Veränderungen des Myokards, die sich auch auf das Endokard ausdehnten (•Abb. 1). Die Läsionen waren von grau-weißer Farbe und grober Textur; sie wirkten narbenartig. Sie waren überwiegend im linken Ventrikel 


\begin{tabular}{|c|c|c|}
\hline & Kardiale Sarkoidose (KS)/idiopathische granulomatöse Myokarditis & Riesenzellmyokarditis (RZM) \\
\hline \multirow[t]{4}{*}{$\begin{array}{l}\text { Klinische } \\
\text { Aspekte }\end{array}$} & $\begin{array}{l}\text { Fehlende extrakardiale Symptome und sogar eine völlig asymptomatische Erkran- } \\
\text { kung bis zum plötzlichen Herztod sind nicht ungewöhnlich; Erstdiagnose häufig } \\
\text { postmortal }[3,4]\end{array}$ & $\begin{array}{l}\text { Wie bei KS häufig Kardiomyopathie mit } \\
\text { Rhythmusstörungen, aber noch schnelle- } \\
\text { rer, fulminanterer Verlauf und schlechtere } \\
\text { Prognose [5] }\end{array}$ \\
\hline & $\begin{array}{l}\text { Wenn kardiale Symptome vor dem Tod auftreten, dann stehen entweder Rhyth- } \\
\text { musstörungen oder Zeichen der Herzinsuffizienz im Vordergrund. Ab Symptom- } \\
\text { beginn meist nur noch relativ kurzes Überleben [3] }\end{array}$ & \multirow{3}{*}{$\begin{array}{l}\text { Seltener als bei KS kommt es zu Synkopen } \\
\text { und plötzlichem Herztod, häufiger werden die } \\
\text { Betroffenen vor ihrem Tod mit Zeichen einer } \\
\text { Herzinsuffizienz symptomatisch [5] }\end{array}$} \\
\hline & Häufiger Reizweiterleitungsstörungen als bei der RZM [5] & \\
\hline & $\begin{array}{l}\text { Todeintritt gehäuft bei körperlicher Ertüchtigung [4], aber auch in Ruhe/im Schlaf } \\
\text { [6]. Insgesamt bezogen auf die Wochen vor dem Tod tendenziell symptomärmer } \\
\text { als die RZM [7] }\end{array}$ & \\
\hline \multirow[t]{4}{*}{ Makroskopie } & $\begin{array}{l}\text { Meist erhöhtes Herzgewicht [3], hypertrophe oder (häufiger) dilatative Kardiomyo- } \\
\text { pathie [5] }\end{array}$ & \multirow[t]{4}{*}{$\begin{array}{l}\text { Ausgedehnte hämorrhagische Bereiche und } \\
\text { Nekrosen [9, p. 201] }\end{array}$} \\
\hline & Manchmal Perikarderguss, selten Perikarditis [8] & \\
\hline & $\begin{array}{l}\text { Weiße, feste Areale, häufig durch das Epi- und/oder Endokard sichtbar [4]. Myokard } \\
\text { mit disseminierten fleckigen, knotig-herdförmigen oder bandartig konfigurierten } \\
\text { Läsionen [3] }\end{array}$ & \\
\hline & $\begin{array}{l}\text { Areale meist in der freien Wand der linken Kammer, in den linksventrikulären Papil- } \\
\text { larmuskeln und im Ventrikelseptum, v. a. im oberen Anteil (AV-Block) [4]! }\end{array}$ & \\
\hline \multirow[t]{5}{*}{ Mikroskopie } & $\begin{array}{l}\text { Nichtverkäsende Granulome mit begrenztem Lymphozyteninfiltrat und fleckiger } \\
\text { Fibrose. Riesenzellen meist im Zentrum von Granulomen [5] }\end{array}$ & $\begin{array}{l}\text { Riesenzellen, aber nicht innerhalb von Granu- } \\
\text { lomen und mit weniger Fibrose in der Nach- } \\
\text { barschaft, vielmehr assoziiert mit diffuser } \\
\text { und ausgeprägter lymphozytärer Infiltration } \\
\text { und myozytären Nekrosen, ferner können } \\
\text { Histiozyten und Eosinophile vorkommen [5] }\end{array}$ \\
\hline & $\begin{array}{l}\text { Granulome sind im Myokard weniger scharf begrenzt und weniger organisiert als } \\
\text { die typischen Sarkoidose-Granulome in Lymphknoten und anderen Organen [3] }\end{array}$ & \multirow[t]{4}{*}{ Epikardiales Fett nicht einbezogen [9, p. 110] } \\
\hline & $\begin{array}{l}\text { Zwei Verteilungstypen von Granulomen im Myokard: Disseminierte Verteilung oder } \\
\text { kontinuierliche Ausbreitung, im Parenchym oder interstitiellen Bindegewebe [3] }\end{array}$ & \\
\hline & Kein Unterschied zur RZM in der Anzahl an Riesenzellen [7] & \\
\hline & $\begin{array}{l}\text { Verschiedene Typen (entsprechend dem Alter der jeweiligen Läsion, können ne- } \\
\text { beneinander vorkommen) [3]: } \\
\text { Exsudativer Typ (Lymphozyten, diffuses Ödem, Histiozyten) } \\
\text { Granulomtyp (Epitheloidzellgranulome mit Riesenzellen und Lymphozyten) } \\
\text { Gemischter Granulom- und Fibrosetyp (atrophe Epitheloidzellgranulome und } \\
\text { Myokardfibrose, manchmal viele Riesenzellen vom Fremdkörper- und vom Langer- } \\
\text { hans-Typ) - häufig! } \\
\text { Fibrosetyp (Myokard durch fibrohyalines Gewebe ersetzt, spärlich Lymphozyten) }\end{array}$ & \\
\hline
\end{tabular}

sichtbar, hier v.a. im herzbasisnahen Anteil des Septum interventriculare, und in den Papillarmuskeln der Trikuspidalklappe. Die Vorhöfe waren nicht betroffen. Neben den blutreichen Organen mit oberer venöser Einflussstauung sowie Lungenödem, Schocknieren und akut gestautem Milzgewebe waren makroskopisch keine dem kardialen Befund ähnlichen Veränderungen an den übrigen Organen sichtbar. Die Lymphknoten waren nicht abnorm vergrößert. Dem makroskopischen Aspekt zufolge wurde ein kardiales Versagen auf dem Boden einer unbekannten (systemischen?) Erkrankung als Todesursache angenommen. Eine Myokarditis wurde vermutet, und histologische Untersuchungen wurden veranlasst.
Die histologischen Untersuchungen zeigten eine ausgedehnte noduläre lymphohistiozytäre Infiltration unter Einbeziehung der noch verbliebenen Kardiomyozyten. Im Hintergrund war eine Fibrose zu erkennen (बAbb. 2). Die Infiltration war nicht auf das Myokard beschränkt, sondern wucherte in das angrenzende Weichgewebe hinein. Es fanden sich auch einige nichtzusammenhängende entzündliche Knötchen. Innerhalb der Infiltration zeigten sich viele Riesenzellen und epitheloide Granulome ohne verkäsende Nekrosen (- Abb. 4b mit CD68 und c mit Siriusrot). Der eher noduläre Aspekt, die epitheloiden Granulome und die Ausdehnung in das perikardiale Weichgewebe sprachen für eine kardiale Sarkoidose vom kombi- nierten granulomatösen und fibrotischen Typ und trotz der vielen Riesenzellen gegen eine Riesenzellmyokarditis.

\section{Diagnose}

Zusammenfassend konnte eine kardiale Sarkoidose (oder idiopathische granulomatöse Myokarditis) als Todesursache festgestellt werden. Infektiöse Myokarditiden sowie ischämische Veränderungen wurden bereits aufgrund der makroskopischen Befunde als unwahrscheinlich erachtet. Die Ausdehnung auf das Endokard, die Verteilung der Läsionen mit fokalem Befall des linken Ventrikels und das narbige Erscheinungsbild der einzelnen Läsionen begründeten den Verdacht auf eine kardiale Sarkoidose (- Tab. 1). Eine Riesenzell- 


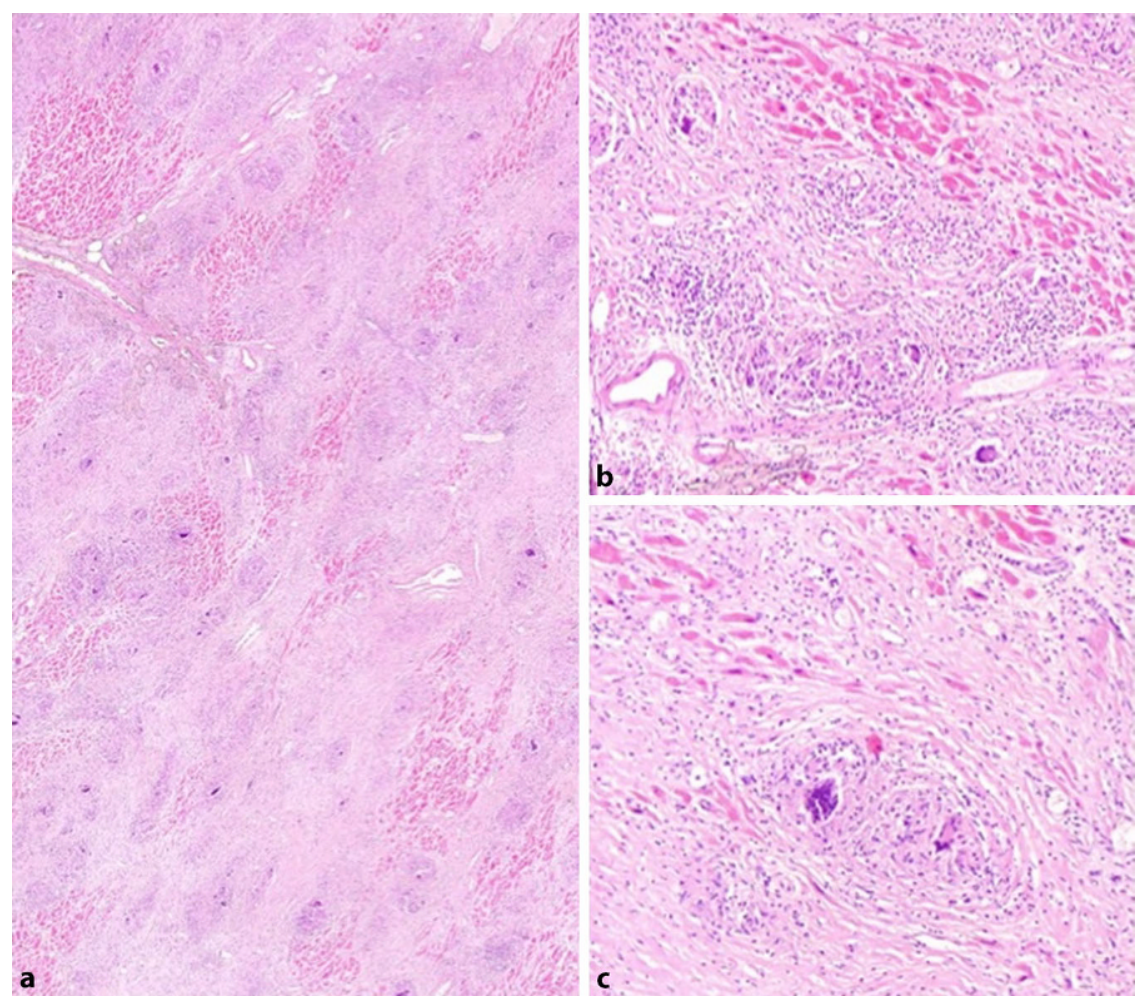

Abb. 3 \ Histologische Darstellung von Fall 2. HE-gefärbtes Gewebe in der Übersicht (a) (Vergrößerung 5x) und Vergrößerung (b,c) (Vergrößerung 10x) mit teilweise spärlicher lymphohistozytärer Infiltration, Riesenzellen, Fibrose und epitheloiden Granulomen, die das Myokard zerstören und eine Fibrose hinterlassen

myokarditis konnte mikroskopisch ausgeschlossen werden. Der klinische Verlauf mit fehlenden extrakardialen Symptomen und Erstmanifestation durch einen plötzlichen Herztod erschien ebenfalls passend zur kardialen Sarkoidose.

Bei den dermatologischen Befunden handelt es sich höchstwahrscheinlich um Zufallsbefunde, die nicht im Zusammenhang mit der todesursächlichen Erkrankung stehen. Es kann jedoch nicht ausgeschlossen werden, dass diese Befunde abgeheilte Hautmanifestationen einer systemischen Sarkoidose sind.

\section{Fall 2}

\section{Anamnese}

Ein 41-jähriger Mann erhielt am Tag seines Todes Besuch von einem Bekannten. In Anwesenheit seines Bekannten kollabierte der Mann unerwartet. Die hinzugerufenen Rettungskräfte unternahmen Wiederbelebungsversuche, die jedoch vor Ort erfolglos beendet werden mussten.

Der Verstorbene trat in der Vergangenheit als Heroinkonsument auf, galt aber als "clean". Zudem seien nicht näher beschriebene "Herzprobleme" bekannt gewesen. Eine von der Polizei vorgelegte Kopie eines Arztbriefes ergab die Diagnosen einer linksbetonten "dilatativen Kardiomyopathie" mit eingeschränkter Herzleistung sowie einer Sarkoidose mit ausgedehnter pulmonaler Beteiligung.

\section{Obduktionsbefund}

Obduziert wurde die Leiche eines stark übergewichtigen Mannes $(182 \mathrm{~cm}, 148 \mathrm{~kg})$ mit ausgedehnter Blutstauung im Kopfund im Halsbereich. Das Herz wog $837 \mathrm{~g}$. Es zeigte eine abgerundete Herzspitze, die durch den linken Ventrikel gebildet wurde. Die Kammerwanddicken maßen rechts $0,6 \mathrm{~cm}$ bzw. links $2 \mathrm{~cm}$. Auf dem Flachschnitt durch die Wand des linken Ventrikels sowie beim Anschneiden der Papillarmuskeln zeigte sich ein diffus weißlich geflecktes Myokard mit verwaschener Gewebszeichnung. In der Wand des rechten Ventrikels fanden sich ebenfalls einige grau-weiße, fleckige Veränderungen. Die Koronararterien waren unauffällig. Es bestanden eine chronische und akute Leber- stauung. Zudem war ein verfestigtes Lungengewebe auffällig, in dem hirseähnliche Knötchen zu tasten waren.

Die chemisch-toxikologischen Zusatzuntersuchungen ergaben keinen Hinweis auf einen akuten Drogenkonsum.

Die histologischen Untersuchungen zeigten wie im Fall 1 einen knotigen Umbau des Myokards mit lymphohistiozytärer Infiltration und vielen Riesenzellen (- Abb. 3). Hier waren die Riesenzellen noch prominenter. Außerdem zeigten sich einige schlecht abgegrenzte epitheloide Granulome ohne verkäsende Nekrosen (• Abb. 4e). In vielen Bereichen war die Entzündung ausgebrannt und hatte fibrotisch umgebautes Gewebe hinterlassen (- Abb. 4f). Auch in diesem Fall waren die Veränderungen nicht vollständig auf das Myokard beschränkt.

Zusammenfassend sprachen auch im Fall 2 die verwaschen abgegrenzten, nichtverkäsenden Granulome und die Fibrose für eine kardiale Sarkoidose vom kombinierten granulomatösen und fibrotischen Typ, die hier teilweise im Übergang zum fibrotischen Typ war. Das noduläre Verteilungsmuster, die epitheloiden Granulome und die Ausdehnung auf das angrenzende Gewebe sprachen trotz der starken Riesenzellkomponente gegen eine Riesenzellmyokarditis.

\section{Diagnose}

DerVerdacht einer Herzbeteiligung bei systemischer Sarkoidose ergab sich bereits am Obduktionstisch aufgrund der typischen Befunde im Myokard beider Ventrikel und in der Lunge. Die Zeichen der Herzhypertrophie und -insuffizienz waren wesentlich ausgeprägter als im ersten Fall. Mikroskopisch wurde der Verdacht auf eine kardiale Sarkoidose bestätigt; eine Riesenzellmyokarditis konnte ausgeschlossen werden (0 Tab. 1).

Die Anamnese mit zuvor bekannter Sarkoidose der Lungen und "Herzproblemen“ vor dem Tod passt zu einer kardialen Sarkoidose, stellt aber im Vergleich zum ersten Fall eine andere Verlaufsform mit einem symptomatischen Verlauf über einen längeren Zeitraum dar. 

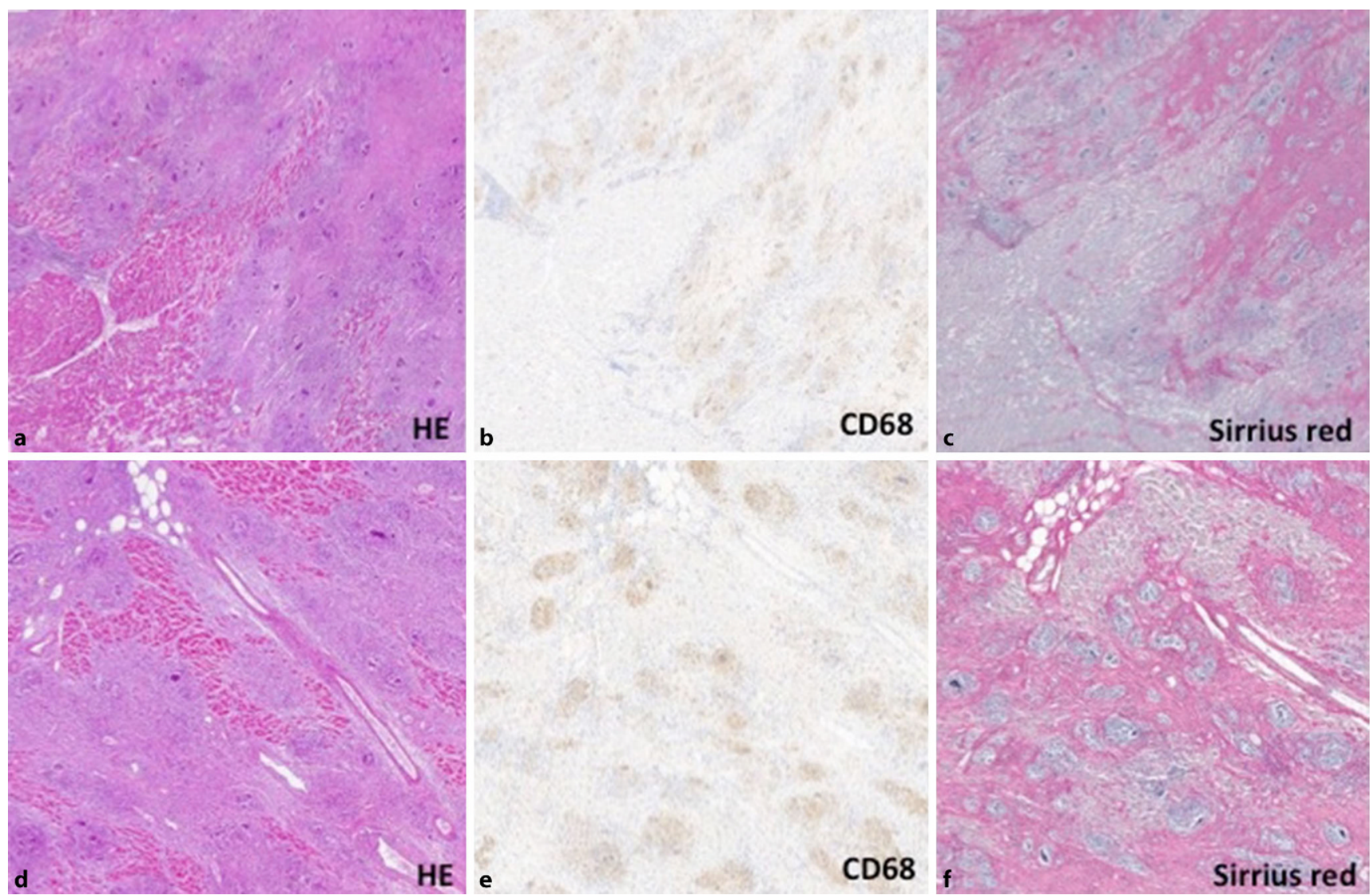

Abb. 4 ム Histologischer Vergleich beider Fälle. HE-Färbung (a,d) (Vergrößerung 5x), Immunhistochemie mit CD68 zur Hervorhebung von Histiozyten und Granulomen (b,e) (Vergrößerung $5 \mathrm{x}$ ) sowie Siriusrotfärbung zur Darstellung der Fibrose $(\mathbf{c}, \mathbf{f})$ (Vergrößerung 5x)

\section{Diskussion}

Die Sarkoidose ist eine systemische Erkrankung unbekannter Ätiologie, die verschiedene Organsysteme betreffen kann und sich histologisch durch nichtverkäsende Granulome auszeichnet. Ein Herzbefall manifestiert sich nicht selten erst durch den plötzlichen Herztod, wie klinisch-pathologische Erhebungen und Einzelfallstudien $[3,4,6,10]$ zeigen. Fehlende extrakardiale Symptome in Fällen tödlich verlaufender kardialer Sarkoidose scheinen nicht ungewöhnlich zu sein. Vereinzelt wird diskutiert, ob sich gar eine negative Korrelation zwischen der Ausprägungsstärke des kardialen und des extrakardialen Befalls behaupten lässt [8]. Auch postmortal erstdiagnostizierte Fälle kardialer Sarkoidose ganz ohne Nachweis sarkoidosetypischer Veränderungen in anderen Organen sind beschrieben [7]. Einerseits ist hier die Möglichkeit einer isolierten kardialen Sarkoidose bzw. idiopathischen granulomatösen
Myokarditis zu erwägen, die bei längerem Überleben vielleicht in eine systemische Sarkoidose übergegangen wäre. Andererseits könnten in einem Teil dieser Fälle extrakardiale Krankheitsherde bei makroskopisch unauffälligen Organen und Geweben der mikroskopischen Untersuchung entgangen sein [4]. Es ist z.B. denkbar, dass befallene Lymphknoten nicht erkennbar vergrößert waren und daher einer histologischen Untersuchung gar nicht erst zugeführt wurden.

Diagnostische Schwierigkeiten können gerade beim Fehlen extrakardialer Befunde in der Abgrenzung der kardialen Sarkoidose von der Riesenzellmyokarditis liegen. Beide Entitäten sind nichtinfektiöse Myokarditiden, die sich in einem plötzlichen Herztod manifestieren können $[6,11]$. Die korrekte Diagnose kann jedoch in Zusammenschau der makroskopischen Befunde und des klinischen Verlaufs mit den Ergebnissen der histopathologischen Untersuchungen gestellt werden ( $\bullet$ Tab. 1).

\section{Fazit für die Praxis}

Die gegenständlichen Fälle demonstrieren eine seltene, aber typische Ursache des plötzlichen Herztodes mit unterschiedlichen klinischen Verläufen. Eine interdisziplinäre Fallbeurteilung kann helfen, auch seltene Erkrankungen in der gerichtlichen Obduktion korrekt zu diagnostizieren. Klinisch sollte bei der Erstdiagnose einer Sarkoidose an eine mögliche Herzbeteiligung gedacht und die Möglichkeit des Auftretens einer solchen auch in der Verlaufsbeobachtung berücksichtigt werden.

\section{Korrespondenzadresse}

\section{Dr. med. Cleo Walz}

Institut für Rechtsmedizin, Universitätsmedizin Mainz

Am Pulverturm 3, 55131 Mainz, Deutschland walz@uni-mainz.de

Funding. Open Access funding enabled and organized by Projekt DEAL. 


\section{Einhaltung ethischer Richtlinien}

Interessenkonflikt. C.-S.Schwarz, C.-A. Weis, A.Marx, T. Germerott und C. Walz geben an, dass kein Interessenkonflikt besteht.

Für diesen Beitrag wurden von den Autoren keine Studien an Menschen oder Tieren durchgeführt.

Open Access. Dieser Artikel wird unter der Creative Commons Namensnennung 4.0 International Lizenz veröffentlicht, welche die Nutzung, Vervielfältigung, Bearbeitung, Verbreitung und Wiedergabe in jeglichem Medium und Format erlaubt, sofern Sie den/die ursprünglichen Autor(en) und die Quelle ordnungsgemäß nennen, einen Link zur Creative Commons Lizenz beifügen und angeben, ob Änderungen vorgenommen wurden.

Die in diesem Artikel enthaltenen Bilder und sonstiges Drittmaterial unterliegen ebenfalls der genannten Creative Commons Lizenz, sofern sich aus der Abbildungslegende nichts anderes ergibt. Sofern das betreffende Material nicht unter der genannten Creative Commons Lizenz steht und die betreffende Handlung nicht nach gesetzlichen Vorschriften erlaubt ist, ist für die oben aufgeführten Weiterverwendungen des Materials die Einwilligung des jeweiligen Rechteinhabers einzuholen.

Weitere Details zur Lizenz entnehmen Sie bitte der Lizenzinformation auf http://creativecommons.org/ licenses/by/4.0/deed.de.

\section{Literatur}

1. Birngruber CG, Hochscheid C, Dettmeyer RB (2020) Unerkannte letale idiopathische Riesenzellmyokarditis - ein Fallbericht. Rechtsmedizin 30:462-465

2. Dettmeyer R (2018) Forensic histopathology, 2. Aufl. Springer, Berlin, Heidelberg, S327-329

3. Matsui Y, Iwai K, Tachibana T et al (1976) Clinicopathological study of fatal myocardial sarcoidosis. Ann NY Acad Sci 278:455-469

4. Roberts WC, McAllister HA Jr., Ferrans VJ (1977) Sarcoidosis of the heart. A clinicopathologic study of 35 necropsy patients (group 1) and review of 78 previously described necropsy patients (group 11). Am JMed 63(1):86-108

5. Blauwet LA, Cooper LT (2013) Idiopathic giant cell myocarditis and cardiac sarcoidosis. Heart Fail Rev 18:733-746

6. Riße M, Verhoff MA, Dettmeyer R (2008) Kardiale Sarkoidose. Rechtsmedizin 18(4):267-268

7. Okura Y, Dec GW, Hare JM et al (2003) A clinical and histopathologic comparison of cardiac sarcoidosis and idiopathic giant cell myocarditis. J Am Coll Cardiol 41(2):322-329

8. Kim JS, Judson MA, Donnino R et al (2009) Cardiac sarcoidosis. Am Heart J 157(1):9-21

9. Connelly AJ, Finkbeiner WE, Ursell PC, Davis RL (2006) Autopsy pathology. A manuel and atlas, 3. Aufl. Elsevier, Philadelphia

10. Jotterand M, Grabherr S, Lobrinus JA et al (2017) Sudden cardiac death and sarcoidosis of the heart in a young patient. Cardiovasc Pathol 28:18-20

11. Matejic D, Schönfeld C, Tsokos M (2010) Plötzlicher Tod bei akuter idiopathischer Riesenzellmyokarditis. Rechtsmedizin 20:275-277

\section{Sudden cardiac death in two cases of cardiac sarcoidosis}

One of the primary objectives of forensic pathology is the resolution of unexpected fatalities. In particular, the identification of rare natural causes of death requires a sound knowledge of typical macromorphological findings as well as initiation and interpretation of appropriate supplementary examinations, such as histological procedures. In order to follow the correct postmortem diagnostic pathways, important differential diagnoses also need to be taken into consideration.

In this article the characteristic macroscopic and microscopic findings of cardiac sarcoidosis (or granulomatous myocarditis) and the differentiation from those of giant cell myocarditis are demonstrated based on two cases. In both cases, the sudden death of a middle-aged adult gave rise to a forensic autopsy, which ultimately led to the diagnosis of cardiac sarcoidosis. Various disease courses are discussed by means of these cases in consideration of relevant scientific publications. With a thorough evaluation of anamnestic and macromorphological findings, the suspected diagnosis of cardiac sarcoidosis can be verified immediately after the autopsy. Histopathological examinations can confirm the diagnosis. This confirms that interdisciplinary casework enables a rapid diagnosis in cases of rare diseases, such as cardiac sarcoidosis.

\section{Keywords}

Giant cell myocarditis · Granulomatous myocarditis · Noninfectious myocarditis · Sudden deaths . Forensic autopsy 